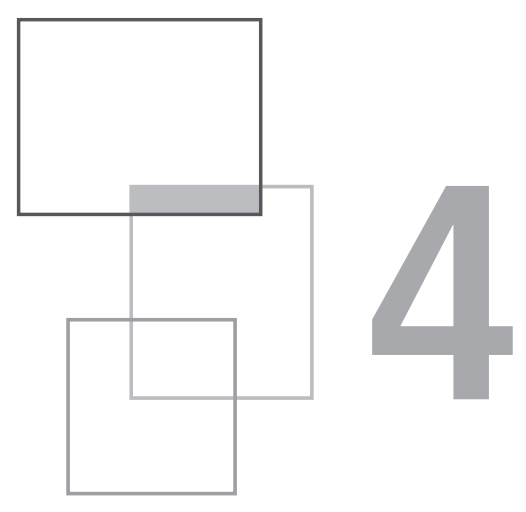

\title{
Les impacts sur la société
}

\subsection{Introduction}

L'énergie (le " feu ") est l'un des quatre éléments fondamentaux de la nature ${ }^{74}$, et c'est par la maîtrise de l'énergie (sous ses formes thermique, mécanique, radiative, chimique...) que la société humaine a pu évoluer, et dépasser notamment le stade de l'esclavage.

L'énergie se conserve (c'est une loi fondamentale de la physique), mais elle change de forme : certaines formes sont plus risquées pour l'homme, d'autres plus impactantes pour l'environnement (voir Partie 2, Chapitre 1).

Comme on l'a vu au Chapitre 3 de cette Partie 2, l'introduction des énergies ${ }^{75}$ renouvelables intermittentes (EnRi) déstabilise l'économie. Nous montrerons dans ce chapitre qu'elles déstabilisent également la société : la CSPE*76 est détournée de son rôle social au profit de subventions pour les nouvelles énergies; les emplois sont délocalisés au profit de l'importation d'aérogénérateurs et de panneaux photovoltaïques ; la durabilité du modèle est mise à mal, en raison du prélèvement de métaux, et des déchets que ces nouvelles énergies génèrent. Enfin, la baisse du taux de retour énergétique réduira les investissements pour la satisfaction des besoins intellectuels, car les besoins primaires de subsistance devront être satisfaits en priorité.

74. Chez les philosophes grecs présocratiques, les 4 éléments fondamentaux sont : la terre, l'air, l'eau, le feu.

75. Selon l'usage vernaculaire, on utilisera le terme «énergie " pour désigner indifféremment des sources d'énergie.

76. Les termes suivis d'un astérisque sont définis en fin de chapitre. 
"Le pire n'est pas toujours sûr " : c'est en prenant conscience des dangers, plutôt que de les cacher " sous le tapis ", que la société française, au sein de l'Europe, parviendra à maitriser la décarbonation de l'énergie, avec les grands défis que sont le chauffage et le transport.

Ce sont ces questions de société que nous nous efforcerons d'aborder dans ce texte.

\subsection{Le développement humain et son impact sur l'environnement}

\subsubsection{L'indice de développement humain (IDH)}

Le Programme des Nations unies pour le développement a créé en 1990 un indice statistique composite pour évaluer le développement - non pas seulement économique - mais humain, l'indice de développement humain (IDH), qui se fonde sur trois critères majeurs : l'espérance de vie à la naissance, le niveau d'éducation, le niveau de vie.

\subsubsection{Développement et consommation énergétique}

La courbe de la figure 1 montre qu'au-delà de 3 Tep*/H/an, l'IDH* n'augmente plus guère : Italie, France, Belgique, États-Unis, Islande sont au même niveau de développement, légèrement supérieur à 0,9 , pour des consommations énergétiques qui varient du simple au quadruple (respectivement : 3,$1 ; 4,9 ; 6,4 ; 8,4$ et $12,3 \mathrm{Tep} / \mathrm{H} / \mathrm{an}$ ).

Entre la France et les États-Unis, le rapport des consommations moyennes d'énergie par habitant est de 1,7 alors que l'IDH de chaque pays est très voisin. Par contre, il apparaît clairement sur la figure 1 qu'en dessous de 1 Tep/H/an, l'IDH est étroitement corrélé à la consommation moyenne d'énergie par habitant.

On définit la sobriété énergétique comme la capacité à atteindre un indice de développement donné pour une dépense énergétique minimale.

\subsubsection{Développement et rejets de gaz à effet de serre $\left(\mathrm{CO}_{2}{ }^{*}\right)$}

La figure 2 montre que, pour un niveau de développement comparable, la France, l'Allemagne le Japon et l'Amérique du Nord émettent respectivement 6, 8, 10, $20 \mathrm{t}\left(\mathrm{CO}_{2}\right) / \mathrm{H} / \mathrm{an}$. Entre la France et les États-Unis, le rapport est de 3,4.

On définit l'efficacité énergétique comme la capacité à atteindre un indice de développement donné pour une émission de gaz à effet de serre (GES, exprimé en équivalent- $\mathrm{CO}_{2}$ ) minimale. 


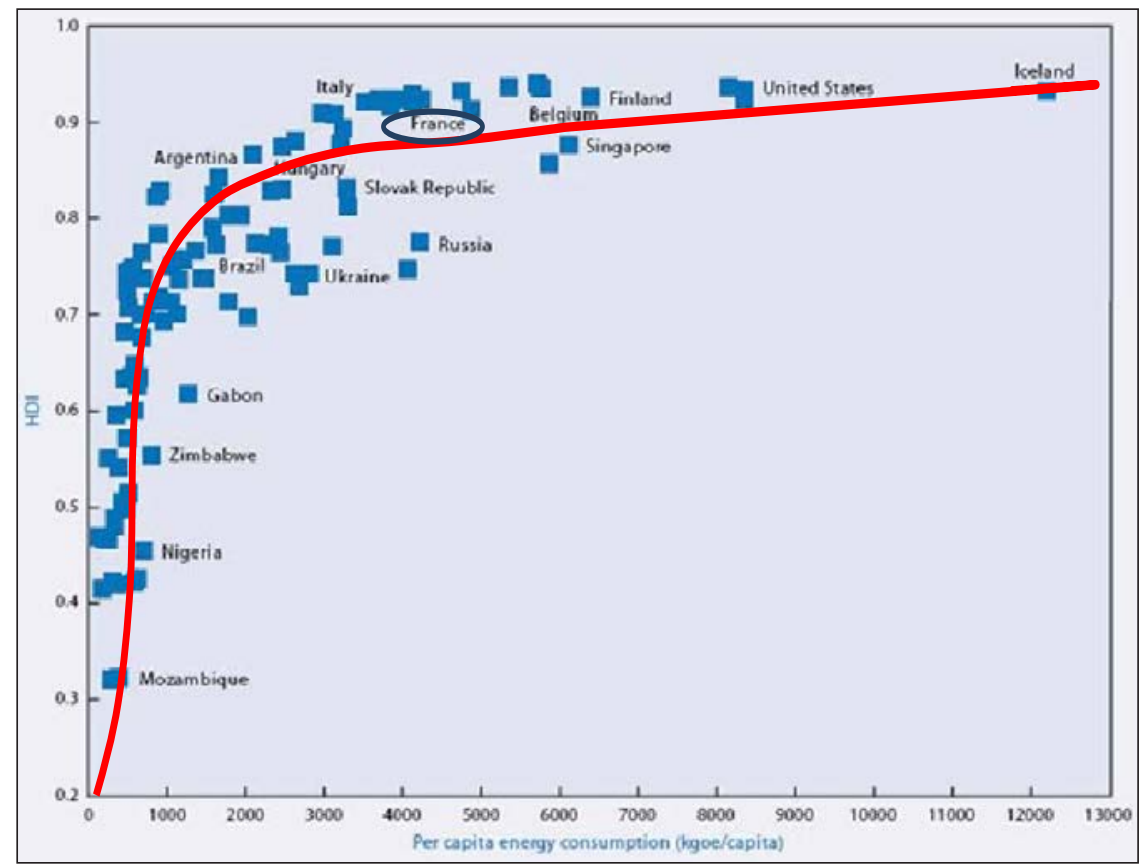

Figure 1 Évolution de l'IDH en fonction de la consommation énergétique (en $\mathrm{kg}$ éq. pétrole par habitant et par an) [1].

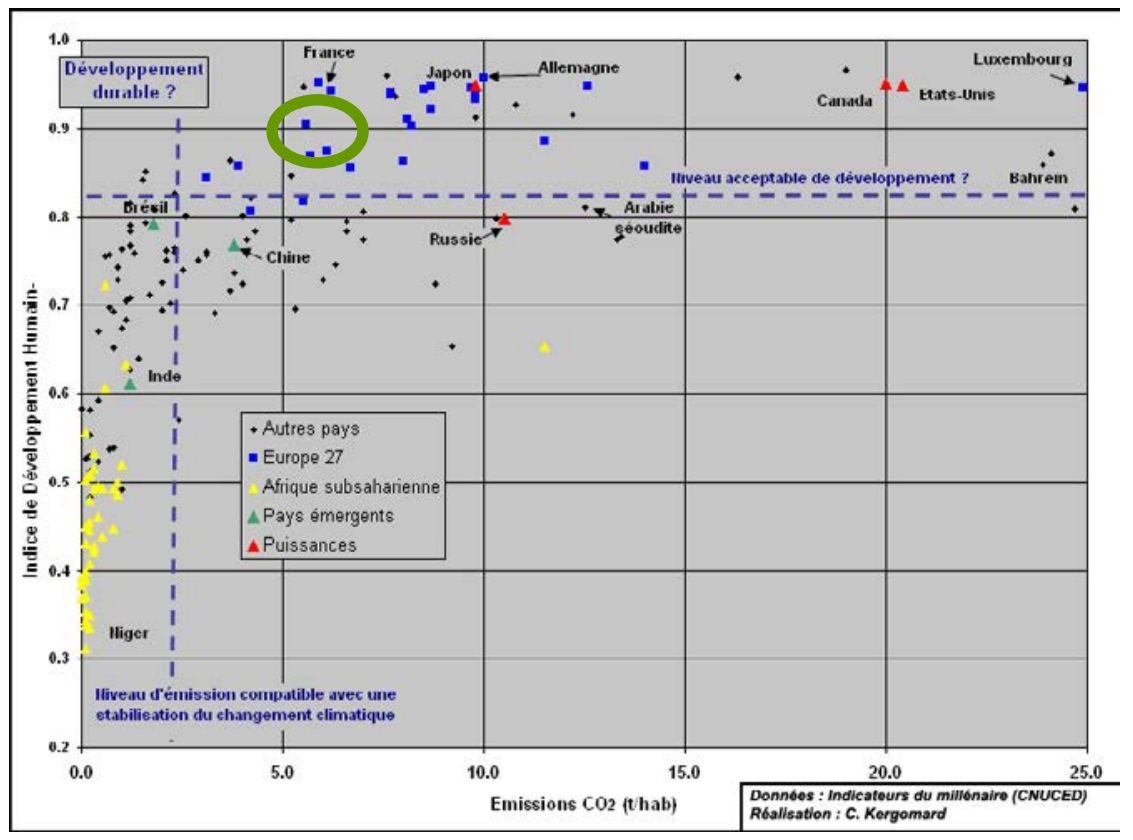

Figure 2 Évolution de l'indice de développement humain (IDH) en fonction des émissions de $\mathrm{CO}_{2}$ (t/H/an) [1]. 
Pour assurer un développement durable sur la planète, il faut que les rejets par habitant et par an soient inférieurs à 2 tonnes de $\mathrm{CO}_{2}$, ce que ne réalisent pour l'instant que les pays sous-développés, mais avec un IDH inférieur à 0,8 .

Soulignons le bon positionnement actuel de la France, parmi les pays développés et avec une population importante, grâce au nucléaire ; dans le cas d'une sortie du nucléaire, les émissions de $\mathrm{CO}_{2}$ augmenteraient de $100 \mathrm{Mt} / \mathrm{an}$ [2].

\subsubsection{Développement et empreinte écologique}

L'empreinte écologique est exprimée ici en nombre d'hectares nécessaires par habitant. La figure 3 montre que pour un niveau de développement comparable, la France, le Canada le Danemark et les États-Unis consomment en espace respectivement 4,$9 ; 7,2 ; 8 ; 9,7$ ha/H. Entre la France et les États-Unis, le rapport est de 2.

Pour assurer un développement durable de l'humanité dans le cadre des ressources limitées de la planète, on estime qu'il faut que l'empreinte écologique soit inférieure à $2 \mathrm{ha} / \mathrm{H}$. Cuba respecte cette limite avec un IDH supérieur à 0,8 .

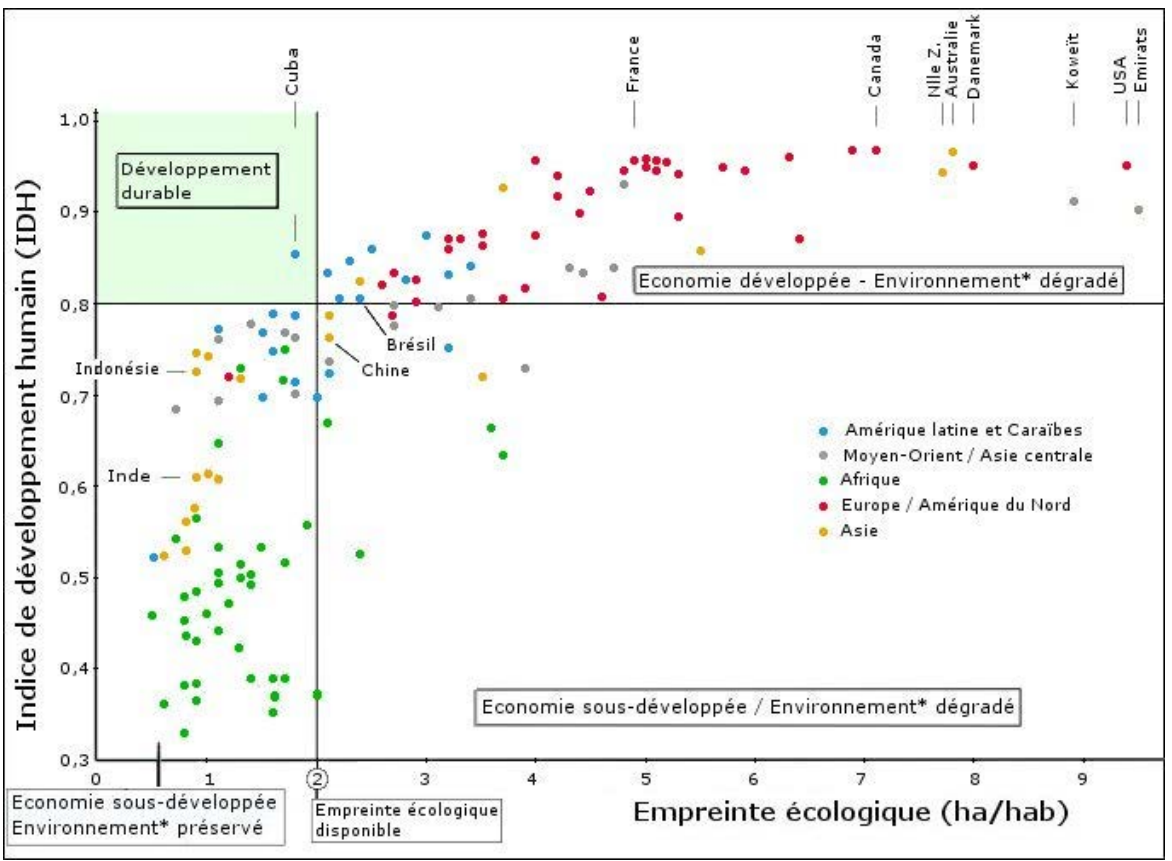

Figure 3 Évolution de l'indice de développement humain (IDH) en fonction de l'empreinte écologique (ha/H) [1]. 


\subsubsection{Pyramide des besoins énergétiques}

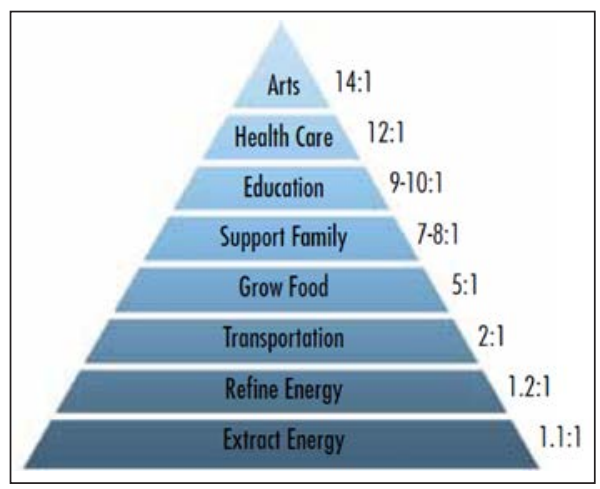

Figure 4 TRE* nécessaires pour satisfaire la pyramide des besoins.

Par analogie à la pyramide des besoins de Maslow, Lambert et al. ont développé une pyramide des besoins en relation avec le TRE* [3].

Par exemple, pour développer un système de santé, une société a besoin d'un TRE de 12, alors que la production alimentaire nécessite un TRE de 5.

Pour satisfaire un échelon, il faut que tous les échelons inférieurs soient satisfaits, d'où l'augmentation du TRE avec les échelons.

Par exemple, pour pouvoir développer l'éducation puis les soins de santé, il faut avant tout avoir suffisamment d'énergie disponible pour l'extraire, la transformer, la transporter et assurer les besoins alimentaires et de logement.

Autrement dit, plus une société mobilise facilement de l'énergie (haute valeur du TRE), plus elle peut consacrer de l'énergie à des activités moins primaires.

\subsubsection{Conclusion}

Les pays développés (IDH de l'ordre de 0,9) consomment beaucoup d'énergie, avec un impact environnemental excessif tant en ce qui concerne les rejets de GES que la consommation d'espace. Pour assurer à tous les humains un développement durable sur la planète, des efforts importants doivent être réalisés pour réduire cet impact sur l'environnement. Cet effort est d'autant plus nécessaire qu'il doit servir d'exemple aux pays en voie de développement. Pour ce qui concerne le $\mathrm{CO}_{2}$, principal GES émis par les activités humaines, il est pratique de décomposer les quantités émises en 4 facteurs :

\begin{tabular}{|c|c|c|c|}
\hline $\begin{array}{c}\text { Favoriser les } \\
\text { énergies } \\
\text { décarbonées } \\
\text { (nucléaire, }\end{array}$ & $\begin{array}{l}\text { Réduire } \\
\text { l'intensité } \\
\text { énergétique } \\
\text { (bâtiments, }\end{array}$ & $\begin{array}{l}\text { Réaliser des } \\
\text { économies } \\
\text { d'énergie } \\
\text { individuelles }\end{array}$ & $\begin{array}{c}\text { Maitriser la } \\
\text { croissance } \\
\text { de la } \\
\text { population }\end{array}$ \\
\hline renouvelables) & $\begin{array}{l}\text { transports, } \\
\text { industrie, } \\
\text { agriculture) }\end{array}$ & & \\
\hline
\end{tabular}




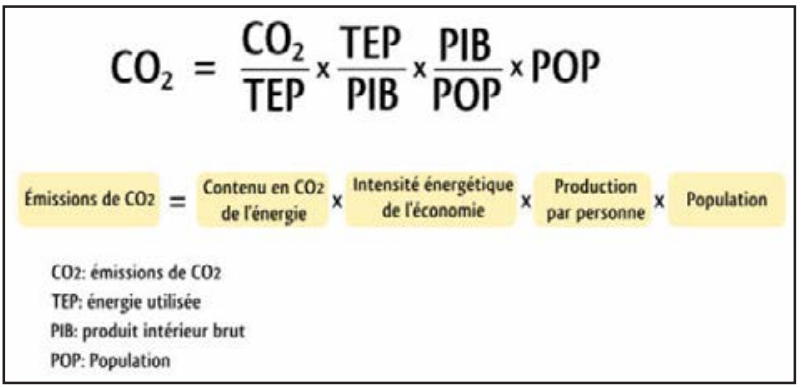

Figure 5 Équation de Yoichi Kaya [1].

En termes d'émission de $\mathrm{CO}_{2}$, le découpage de facteurs de rejet selon cette égalité, dite " équation de Yoichi Kaya " (Figure 5) permet d’identifier les paramètres d'action : favoriser les énergies décarbonées, réduire les dépenses énergétiques par l'efficacité des systèmes et des procédés, viser la sobriété individuelle et collective (en réduisant les besoins). Mais tous ces efforts seront vains sans une maîtrise de la croissance de la population mondiale.

En dehors de l'industrie et de l'agriculture, les grands postes de consommation d'énergie en France sont : la production d'électricité, les transports et le chauffage (voir Partie 1, Chapitre 2). Transports et chauffage relèvent également de l'efficacité et de la sobriété, ils seront donc traités séparément dans ce document. En matière de décarbonation de l'énergie, nous traitons donc ci-dessous les aspects liés à la production d'électricité.

\subsection{Les enjeux sociétaux liés à la décarbonation de l'électricité}

Le sujet de l'impact des énergies sur l'environnement est traité dans ce document plus complètement dans la Partie 2, Chapitre 1. On évoque ci-dessous la façon dont la société les perçoit en 2017.

\subsubsection{Les interrogations vis-à-vis du nucléaire}

La France a développé depuis le deuxième choc pétrolier (1973) une production d'électricité décarbonée à base de nucléaire, mais en 2017, il subsiste des questions qui font encore débat :

1) L'évacuation après un accident grave ${ }^{77}$ : quitter la terre de ses aïeux pour un agriculteur ou un viticulteur est un crève-cœur ; le retour pose des problèmes de

77. On définit comme accident grave un accident provoquant une fusion du cœur. 
réorganisation des structures sociales abandonnées (mairie, école, hôpital, etc.), comme le montre la gestion post-accidentelle de la zone évacuée de Fukushima. La réponse est dans la conception des réacteurs (les réacteurs de Génération III comme l'EPR sont conçus pour confiner les produits radioactifs, même en cas d'accident grave) et les mesures d'intervention mises en place en France après l'accident de Fukushima, afin d'éviter l'évacuation durable de la population avoisinante :

- noyau dur, permettant d'assurer les fonctions de sûreté essentielles (refroidissement du cœur, confinement des produits radioactifs), en cas d'accident majeur touchant l'ensemble du site ;

- force d'action rapide nucléaire, permettant d'assurer la présence permanente de personnel qualifié pour gérer la situation accidentelle jusqu’au retour à l'état sûr.

L'efficacité de ces mesures de prévention, à la fois techniques et organisationnelles, n'aura, espère-t-on, jamais à être démontrée. Des exercices de crise impliquant les populations avoisinantes, y compris au débriefing, peuvent contribuer à regagner la confiance de la population vis-à-vis de la prévention et de la gestion des crises.

2) La gestion des déchets nucléaires. Contrairement aux mensonges des opposants qui ressassent "il n'y a pas de solution ", il est rappelé en Partie 2, Chapitre 2 que la gestion des déchets nucléaires est assurée dans le cadre de la loi de 2006, sans aucun impact sur l'environnement ni sur les populations. Soulignons dans ce domaine les spécificités françaises : 1 ) les combustibles usés sont retraités, selon les principes du développement durable, alors qu'à l'étranger ils sont considérés comme déchets ; 2) les déchets de très faible activité (TFA) sont triés, conditionnés et évacués au travers de filières dédiées (Centre de stockage de l'Aube, CSA); alors qu'à l'étranger ils sont remis dans le circuit industriel banal si la mesure de leur radioactivité est inférieure au seuil de libération.

\subsubsection{L'acceptation des énergies alternatives}

Les éoliennes sont bruyantes, elles émettent des éclairs lumineux (pour se signaler de la circulation aérienne). Le débat porte notamment sur l'éloignement des habitations (500 m, ou $1000 \mathrm{~m}$ au minimum pour les plus hautes) (voir Partie 2, Chapitre 2).

Les panneaux photovoltaïques enlaidissent les toitures, et ne sont favorisés en France que s'ils sont intégrés au bâti. Comme leur densité énergétique par unité de surface est faible (par an, de l'ordre de $200 \mathrm{kWh} / \mathrm{m}^{2}$ soit $\left.2000 \mathrm{MWh} / \mathrm{ha}\right)^{78}$, les centrales photovoltaïques ne peuvent être installées que sur des territoires sans valeur agricole ni touristique.

Les hydroliennes occupent les fonds des chenaux maritimes où les courants sont les plus forts, et donc a priori les plus poissonneux. Le partage du milieu maritime

78. Au prix de $50 € / M W h$, la production serait valorisée à $1000 € /$ ha/an, ce qui est faible. 
soulève des questions, que ce soit pour les hydroliennes ou pour les éoliennes en mer (zones de pêche et trafic maritime).

La géothermie est une source peu visible et apparemment peu polluante. Cependant, la géothermie profonde met en ouvre des techniques de fracturation de la roche qui sont en France interdites pour l'exploitation du gaz de roche-mère. La géothermie de surface ne peut être mise en place que pour des constructions nouvelles. En bord de mer, la thalassothermie permet de climatiser les espaces résidentiels en fournissant une eau tempérée l'hiver et fraîche l'été, qui permet d'utiliser des pompes à chaleur eau-eau avec une grande efficacité.

En matière de transport, les solutions évoquées sont les batteries électriques et l'hydrogène. Les batteries, dont la durée de vie est limitée (de l'ordre de 3 ans - ou un millier de recharges), nécessitent un traitement de recyclage pour ne pas augmenter les déchets. L'hydrogène, quant à lui, est un gaz dangereux ${ }^{79}$, certes utilisé industriellement, mais qui ne pourra être distribué au grand public qu'après une sensibilisation aux risques.

\subsubsection{L'exemple des éoliennes}

L'étude sociologique [4] réalisée en Bretagne par le ministère de l'Environnement et du Développement durable fait apparaître le doute, voire l'opposition, au développement des éoliennes. Doutes sur leur utilité, dans la région de Plovezet près de Penmarc'h (Finistère Sud), quand les éoliennes ne sont pas toutes en mouvement alors qu'il y a du vent. Sentiment de saturation pour les habitants de Mohon, encerclés par trois parcs éoliens, dont les éclairs lumineux ne leur laissent pas de répit, même la nuit. La seule acceptation, sans être inconditionnelle, est celle des habitants du Méné, dont les éoliennes s'inscrivent dans le cadre d'une opération plus vaste d'autoconsommation énergétique, avec une forte implication des élus locaux.

Alors que les "rurbains " fraîchement implantés, venus chercher le calme de la nature, sont "vent debout " contre les éoliennes, les ruraux sont plus favorables à l'implantation de ces nouvelles machines : ils ont tellement vu leur environnement changer depuis les années cinquante!

De fait, ces formes d'énergie diluées et nécessitant du terrain, comme les éoliennes et les panneaux $\mathrm{PV}^{*}$, peuvent constituer un apport complémentaire de revenus pour les paysans, qui souffrent de la concurrence européenne.

Curieusement, alors que des manifestations énormes sont organisées contre l'implantation de centrales nucléaires (Creys-Malville, Plogoff), de réservoirs pour l'irrigation (Sivens), ou de sites de transport (aéroport de Notre-Dame-des-Landes), la privatisation de la mer, espace public commun à tous, ne fait l'objet d'aucune protestation : ce qui montre que l'acceptation d'une nuisance est très relative, et dépend beaucoup de l'activisme des associations relayées par les médias, parfois très complaisants.

79. Il explose avec l'oxygène de l'air moyennant un faible apport énergétique ; voir notamment les explosions des structures des réacteurs de Fukushima. 


\subsubsection{Les énergies nouvelles et la consommation des ressources naturelles}

On note par exemple que les groupes turbo-alternateurs des centrales nucléaires fonctionnent 4 fois plus longtemps à la puissance nominale que les alternateurs des éoliennes, et ont une densité de courant dans le cuivre 4 fois supérieure. Donc, pour une production donnée, la quantité de cuivre immobilisée dans les éoliennes est 16 fois supérieure à celle immobilisée dans le nucléaire.

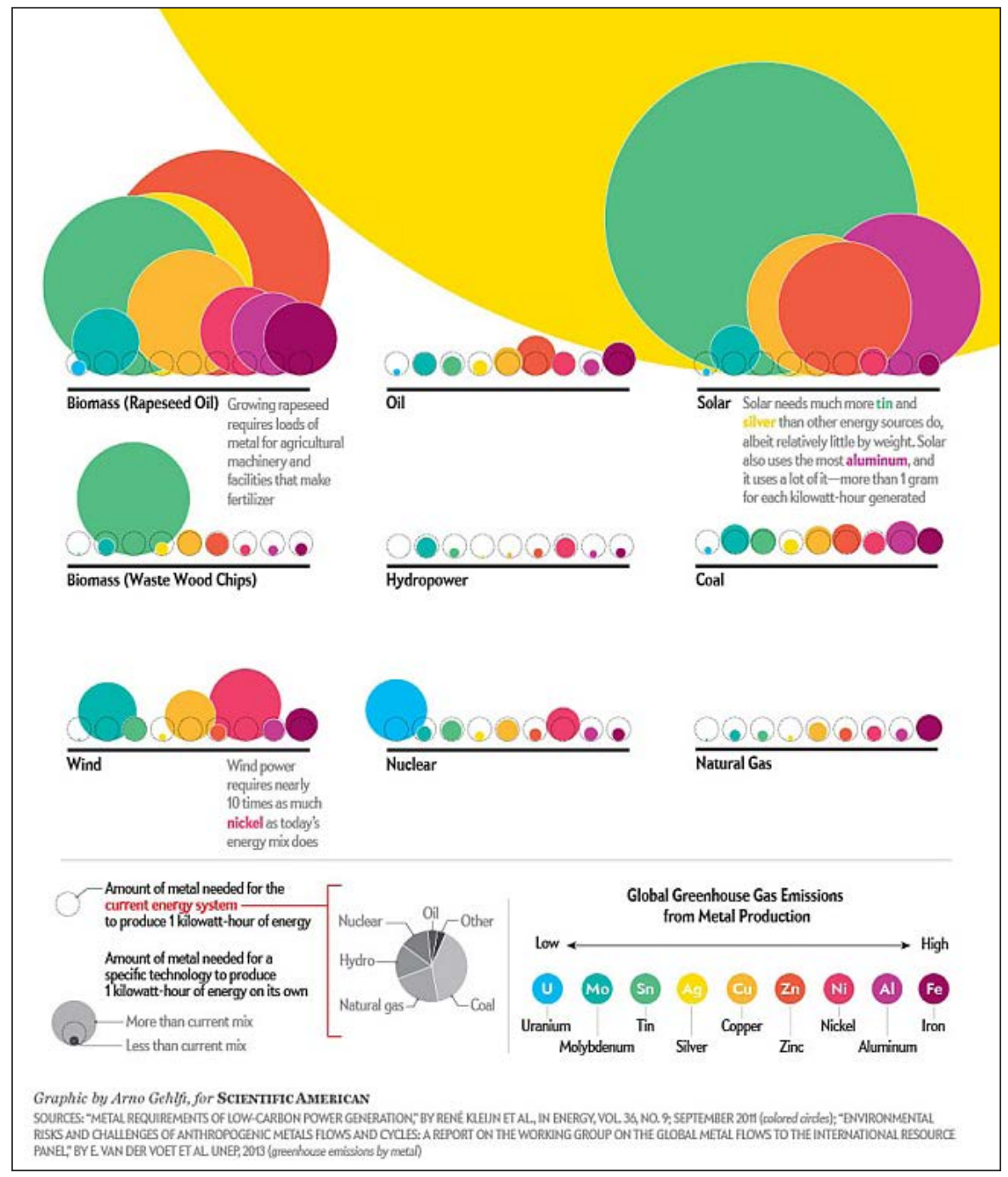

Figure 6 Besoins en matières premières pour développer le programme mondial d'énergies renouvelables [6]. 
De même, les aimants permanents des éoliennes nécessitent des terres rares (néodyme, erbium, dysprosium) qui se trouvent principalement en Chine. Au résultat, les éoliennes sont maintenant principalement importées de Chine, ce qui impacte la balance commerciale de la France. À noter que la Banque mondiale s'est récemment émue que le développement des énergies renouvelables nécessitera d'extraire d'énormes quantités de minerais, ce qui constituera un impact majeur sur l'environnement [5] (voir figure 6). On peut simplement espérer que pour les générations suivantes de matériels, une grande quantité des matières utilisées dans les équipements de première génération seront recyclées, mais les procédés restent à développer, notamment pour le photovoltaïque, le plus gros consommateur de métaux (argent, notamment) par MWh produit.

À noter que dans le nucléaire, la France a fait le choix du cycle fermé du combustible, contrairement à d'autres pays (notamment les États-Unis), en mettant en œuvre le traitement et le recyclage du combustible usé. Cette stratégie permet potentiellement de réduire de $25 \%$ les importations d'uranium naturel (en pratique, la totalité de l'uranium issu du traitement n'est pas réutilisée de sorte que l'économie de matière première est aujourd'hui de l'ordre de $17 \%$ ). De plus, on récupère ainsi l'uranium-238 et le plutonium-239, qui seront les combustibles des réacteurs de $4^{\mathrm{e}}$ génération, offrant à notre pays une réserve de combustible de plusieurs centaines d'années. Ainsi, ce qui est ailleurs considéré comme des déchets de très haute activité est valorisé en France dans le cadre d'une vision durable de la technologie nucléaire.

\subsubsection{Le développement des " smart grids "; la fronde anti-Linky}

Afin d'accepter sur le réseau de distribution basse tension l'électricité produite par les petits producteurs, il est nécessaire de mailler le réseau, comme l'est le réseau de transport haute tension, et de bien connaître à tout instant les sources et les puits de consommation d'électricité : c'est ce qu'on appelle les "smart grids", ou réseaux communicants. En France, le système se construit à partir de l'élément de base que constitue le compteur individuel électronique communicant dénommé Linky, qui permet de connaître à tout instant la consommation - et éventuellement la production - des ménages.

Mais la communication vis-à-vis du public du gestionnaire de réseau Enedis sur cette politique de changement de compteurs fait craindre aux utilisateurs une intrusion dans leur vie privée, ce qui entraîne une fronde dans les petites communes ${ }^{80}$. En Europe, la Grande-Bretagne et l'Italie sont équipés de tels compteurs, mais pas l'Allemagne ni la Belgique : car pour la gestion du réseau, il n'est nul besoin de descendre à ce niveau de granulométrie, l'information gérée au niveau du transformateur

80. Fronde lancée par Stéphane Lhomme, connu par ailleurs comme activiste antinucléaire. 
de quartier suffit. Ceci illustre l'utilisation dévoyée d'arguments énergétiques à des visées commerciales autres que la simple réduction de la consommation.

\subsubsection{Conclusion : la perception du risque est relative}

Dans les années cinquante, il n'était pas de semaine sans qu'un coup de grisou fasse en France des blessés, voire des morts. Aujourd'hui, grâce à la prévention des accidents au travail, la fréquence des accidents a notablement diminué, mais la peur, qui sert à motiver l'intérêt du public, continue d'être instillée par les médias sans référence aux risques réels.

Il serait souhaitable de développer des échelles de risque objectives et indépendantes des intérêts commerciaux : ce serait à l'État de montrer l'exemple, en faisant appel à des comités de sages, issus par exemple des académies, et de défendre leurs évaluations devant le public. Car les hommes (et femmes) politiques sont en général trop dépendants de leur électorat local pour se placer à contre-courant de l'opinion publique.

\subsection{Les conséquences sociales de la transition énergétique}

Un développement humain durable n'est pas nécessairement compatible avec la société consumériste actuelle, qui sous-tend le comportement des agents économiques, producteurs et consommateurs. Il faudra des crises importantes pour faire prendre conscience à la population de la nécessité d'un changement de paradigme : crise technique, avec des black-out* comme en Californie et en Australie ; crise financière, avec la destruction de valeur boursière des sociétés de production d'électricité ; crise environnementale, avec des inondations suite à des ouragans sur les terres, ou des tempêtes accompagnant la montée des niveaux des mers ; ou des crises de pollution extrême, comme le smog de Londres qui a tué des milliers de personnes en 1952.

\subsubsection{L'impact sur les emplois}

L'étude [2] fait apparaître une certaine compensation en nombre : les emplois gagnés dans le domaine des énergies renouvelables compenseraient les emplois perdus dans l'électronucléaire. Il faut cependant souligner les différences en termes qualitatifs : moindre technicité des emplois, localisation de la production à l'étranger (seul le montage étant réalisé sur le territoire national), remplacement d'emplois de 
l'économie marchande par des emplois subventionnés. Jacques Percebois ${ }^{81}$ exprime les mises en garde suivantes :

1 On ne lâche pas la proie pour l'ombre en sacrifiant une filière énergétique française d'excellence, comme le nucléaire.

2 Les seuls emplois industriels durables sont ceux créés par une activité non subventionnée, en visant le marché mondial, sinon on détruit ailleurs plus d'emplois qu'on n'en a créés.

3 Les transitions que cette nouvelle donne industrielle implique devront être accompagnées de manière anticipée par une réorientation de l'effort de formation des professions concernées.

4 On ne crée pas une filière industrielle en la fondant prioritairement sur le marché intérieur. Mais photovoltaïque et captation-stockage du carbone (CSC) sont des technologies appelées à un fort développement mondial, il serait absurde de ne pas les considérer si l'industrie française peut y exceller (ce qui est le cas).

\subsubsection{L'impact sur le niveau de vie}

Lénergie est un poste important de consommation des ménages puisqu'il représente en moyenne 8,4\% de leurs dépenses en 2006 : 4,8 \% pour leur résidence et 3,6 \% pour leur moyen de transport individuel. En $2006^{82}$, chaque ménage débourse ainsi en moyenne $2300 €$ par an pour payer l'énergie de son logement, et le carburant. À noter cependant que la part allouée aux dépenses d'énergie dans le budget d'un ménage est près de deux fois plus faible dans l'agglomération parisienne $(5,7 \%$ en 2006) qu'en zone rurale (11,3\%).

Par ailleurs, un ménage dont le logement est chauffé au fioul dépense environ $28 \%$ de plus au mètre carré qu'un même ménage ayant choisi l'électricité, du fait notamment des normes d'isolation auxquelles le bâtiment doit alors satisfaire. Le Centre d'analyses stratégiques a fait une étude dont il ressort que la sortie du nucléaire pourrait amener une perte de $1 \%$ sur le pouvoir d'achat des ménages et une perte de $0,9 \%$ sur le PIB* [Réf. 2].

\subsubsection{L'impact sur les déplacements}

Comme on l'a vu dans la Partie 1, Chapitre 2, le transport représente $31 \%$ de la consommation d'énergie finale en France. La décarbonation des transports passe actuellement par le recours à l'électricité, ou à un carburant non carboné qu'est l'hydrogène.

81. Jacques Percebois, Doyen honoraire de l'université de Montpellier, dirige le Centre de recherche en économie et droit de l'énergie (CREDEN).

82. Ces données, qui datent de plus de 10 ans, sont données à titre d'illustration. Les ordres de grandeur n'ont guère évolué depuis 10 ans. 
La motorisation électrique des transports en commun date de plus d'un siècle. Le retour à une plus grande part pour les transports publics dans les déplacements est un moyen de réduire globalement les importations de combustible carboné. Le mode de vie actuel est néanmoins basé sur plus de déplacements et de fluidité pour répondre à des emplois plus diversifiés et fractionnés, ce qui induit des transports individuels fréquents.

Une première mesure pour réduire les déplacements serait de développer la télématique et notamment le télétravail, d'une part pour en réduire le nombre, d'autre part pour pouvoir en maîtriser les horaires hors des pics de circulation. Un investissement prioritaire au plan national est donc de multiplier les lignes informatiques à haut débit.

Par ailleurs, outre la pollution chimique et sonore qu'ils induisent, une nuisance importante des véhicules individuels est la surface qu'ils prennent dans les villes, tant dans les avenues et les voies rapides que pour leur stationnement. Les solutions de partage, en attendant que se généralise la conduite autonome, permettent de limiter la place accordée à la voiture, et de la restituer aux modes doux (piétons, vélos) et aux deux-roues électriques. Dans l'immédiat, il est facile de réaliser une économie d'énergie à un coût modéré : la réduction de la vitesse de $10 \mathrm{~km} / \mathrm{h}$ sur autoroutes et routes nationales amènerait plus de $10 \mathrm{TWh}$ d'économies d'énergie.

En conclusion, la décarbonation des transports ne pourra se faire qu'avec une transformation fondamentale de l'image mentale qu'on a des déplacements : abandon du " tout bagnole ", avec la liberté d'horaires et la jouissance d'un objet personnel qu'elle induit. L'aversion contre la pollution et le mal vivre dans les bouchons seront des incitations puissantes pour changer de paradigme.

\subsubsection{L'impact sur le chauffage et l'isolation des bâtiments}

\subsubsection{L'état des lieux}

Comme cela a été décrit dans la Partie 1, Chapitre 2, le résidentiel-tertiaire représente $42 \%$ de la consommation d'énergie finale en France. Il comprend principalement le chauffage et le refroidissement (pour $62 \%$ ), l'eau chaude sanitaire et la cuisson (pour $16 \%$ ) ; le reste concerne la ventilation et l'éclairage.

Le chauffage électrique (13\%) est rentable pour des bâtiments bien isolés, car il ne requiert pas d'investissement à la construction. Le chauffage au bois est également significatif $(21 \%)$, notamment dans les campagnes où la ressource est quasiment gratuite. Le chauffage au pétrole reste à un niveau significatif (19\%) : compte tenu du coût de l'investissement, ce n'est qu'à l'occasion d'une panne entraînant un changement de chaudière qu'il est rentable de remplacer le pétrole par du gaz, ou par une pompe à chaleur. 


\subsubsection{La précarité énergétique}

C'est une réalité qui touche plus de 3 millions de ménages : 3,8 millions en précarité monétaire (ménages consacrant plus de $10 \%$ de leur revenu disponible au chauffage de leur logement) ; 3,5 millions qui ont souffert du froid pendant plus de 24 heures. Quelque 621000 ménages souffrent aujourd'hui du froid et dépensent plus de $10 \%$ de leur revenu pour chauffer leur logement [Réf. 2]. C'est donc ces ménages qu'il faut aider en priorité pour isoler leur logement et remplacer leur mode de chauffage par un système plus efficace.

\subsubsection{Le chauffage électrique, une spécificité française}

Les antinucléaires n'ont de cesse de critiquer le chauffage électrique, alors que les autres pays européens se chauffent principalement au gaz : pertes thermiques du rendement de production d'électricité (environ $33 \%$ ), pic de consommation le soir après le retour au foyer. En fait, le chauffage électrique est modulable - voire effaçable - dès lors que la tarification est incitative et l'isolation du logement satisfaisante. Ce n'est donc pas fatalement un usage de pointe mais au contraire un moyen de stocker de l'électricité intermittente à l'échelle de la journée, sous forme d'eau chaude sanitaire, et en utilisant des radiateurs à inertie.

\subsubsection{L'isolation des locaux et l'investissement en efficacité thermique}

L'isolation des locaux est constamment rappelée comme la panacée, tant pour faire des économies d'énergie que pour favoriser des emplois non délocalisables. L'étude réalisée par l'UFE ${ }^{83}$ met en avant les priorités (isolation des combles, et des parois par l'extérieur ; remplacement des chaudières au fioul par des chaudières à condensation à gaz, ou des pompes à chaleur). Au total, ce sont environ $33 \mathrm{TWh}$ qui pourraient être épargnées grâce à des investissements rentables (TRI* \# 7 ans), soit $6 \%$ de la production d'électricité annuelle ou $2,7 \%$ de la consommation d'énergie finale, mais avec un coût du $\mathrm{CO}_{2}$ épargné très lourd : $2000 €$ par tonne, alors que les taux d'échange actuels sont de quelque $10 € /$ tonne!

Sur le plan du comportement, il faut tenir compte que vivre dans des bâtiments fermés et dotés d'une aération mécanique nécessite un apprentissage, qui peut atteindre plusieurs années dans des bâtiments publics comme les écoles.

\subsubsection{Structuration de la filière et formation du personnel}

Pour 2,3 Md€ de crédits d'impôts consentis par l'État, seulement 10000 rénovations globales environ ont été réalisées, principalement par effet d'aubaine. Car le particulier souhaitant rénover doit s'improviser maittre d'œuvre et coordonner de nombreux corps de métier qu'il ne connaît pas. Seule une politique publique de

83. Union française de l'électricité ; http://ufe-electricite.fr/IMG/pdf/ufe_etude_1_.pdf. 
rupture, basée sur un signal réglementaire clair, et organisée sur une période longue, pourra permettre d'atteindre les objectifs annoncés :

- 125 TWh d'économies d'énergie ;

- $25 \mathrm{Mt} / \mathrm{an}$ de baisse des émissions de $\mathrm{CO}_{2}$;

- 450000 créations d'emploi directs et indirects sur une période de 15 ans, et pérennisés sur la période 2030-2050 [7].

L'enjeu réside dans la structuration d'une filière encore très morcelée et artisanale, dont la segmentation ne permet pas la bonne circulation des compétences. Car pour trouver des clients, il faut :

1) être capable d'annoncer un résultat (dépense énergétique épargnée, temps de retour sur investissement) ;

2) prouver qu'on pourra le tenir (contrôle qualité, coûtenance, personnel formé et compétent). C'est donc un gros effort de formation que la nation devra réaliser, mais c'est un investissement rentable à terme pour toute la vie économique du pays.

\subsubsection{Du chauffage individuel au chauffage collectif}

Pour l'instant, contrairement aux idées propagées, les emplois créés par l'isolation thermique des bâtiments sont peu nombreux (30 000, soit $1 \%$ des sans-emploi en France en 2016) pour un coût de $95000 €$ par emploi créé. En ce qui concerne l'impact des actions d'efficacité énergétique sur la balance commerciale nationale, seules les filières "solaire thermique " et "bois énergie " présentent un solde positif. Les autres contribuent à la dégradation de la balance commerciale française : les chaudières à condensation (pour $173 \mathrm{M} €$ ) et l'isolation thermique des parois opaques et vitrées (pour $213 \mathrm{M} €$ ) sont importées des pays du nord de l'Europe, les pompes à chaleur (pour $479 \mathrm{M€)}$ ) sont importées d'Asie.

Dans l'évolution actuelle, la rénovation des bâtiments pousse au chauffage collectif, du fait de l'importance des investissements (géothermie) ainsi que pour limiter la pollution : les cheminées individuelles non filtrées des chauffages à bois polluent autant en particules fines que des moteurs diesel, et actuellement l'investissement ne peut être amorti et optimisé dans son fonctionnement que par le chauffage collectif. Il s'agit là d'une inversion de mentalité qui prendra du temps à se concrétiser.

\subsection{Conclusions}

La nature du mix énergétique n'est pas sans conséquences pour la prospérité et la cohésion d'un pays. En effet, les erreurs en la matière sont longues à réparer compte tenu des investissements en jeu. Il est donc important de déterminer si un mix énergétique envisagé pour 2050 est bien accessible et approprié.

Un scénario de mix énergétique peut être apprécié selon les critères suivants : 1) la préservation de l'environnement ; 2) la sécurité d'approvisionnement ; 
3) la compétitivité ; 4) la flexibilité. Car un mix énergétique en 2050 ne doit pas être considéré de façon indépendante du chemin pris pour l'atteindre et des hypothèses sous-tendant sa réalisation : une vision énergétique doit privilégier la réversibilité.

\subsubsection{Acceptation et appropriation}

Si la concertation est un point important dans l'acceptation des technologies, des incitations économiques ainsi que des structures de financement plus participatives permettent de redéfinir le lien entre la population et les technologies, en passant d'une notion d'acceptabilité à celle d'appropriation. L'Allemagne et le Danemark en fournissent de bons exemples : la plupart des installations EnRi* y sont détenues par des citoyens (proches des installations), ce qui tend à favoriser l'acceptation des technologies telles que l'éolien sur le territoire. $50 \%$ des investissements dans les énergies renouvelables ont été réalisés par des individus ou des agriculteurs.

\subsubsection{Des critères autres qu'économiques}

Les bénéfices des énergies renouvelables ne peuvent pas tous être quantifiés : détente internationale par une moindre compétition pour les ressources fossiles, impossibilité de détournement de ces énergies à des fins militaires, mise en valeur décentralisée propice à soutenir la démocratie locale [8].

\subsubsection{Dédiaboliser le nucléaire}

Les opposants au nucléaire mettent en avant deux arguments : le risque d'accident grave, et la production de déchets. Si le projet CIGEO répond techniquement au stockage géologique des déchets de haute et moyenne activité à vie longue, les catastrophes de Tchernobyl (1986) et de Fukushima (2011) ont ébranlé la confiance vis-à-vis du risque d'accidents graves. Il est important de souligner que la sûreté ne relève pas seulement de solutions techniques, mais s'appuie avant tout sur une capacité d'organisation rigoureuse. Dans ce domaine, l'État ne valorise pas suffisamment l'évolution importante de l'organisation de la sûreté nucléaire accomplie en France depuis les années 1990, qui s'est traduite par la loi relative à la transparence et à la sécurité en matière nucléaire (TSN) votée en 2006.

Au contraire, le nouveau président élu en 2012 a annoncé un objectif irréaliste, à savoir de baisser à $50 \%$ la part de la production électrique d'origine nucléaire à l'horizon 2025, alors qu'il est évident que cela ne pourra pas se faire en assurant le complément avec des sources d'énergie non maîtrisables, sauf en cas de crise économique profonde et durable, ou à condition d'importer des hydrocarbures de pays politiquement instables, en accroissant le déficit commercial et en polluant davantage en $\mathrm{CO}_{2}$. Par ailleurs, ramener le nombre de réacteurs à une quarantaine ne diminue pas significativement le volume de déchets à traiter. Mais le nucléaire ne 
peut se maintenir que dans une société qui a confiance en elle-même. La maîtrise d'autres activités complexes à fort risque, dans l'aéronautique et le spatial, peut servir d'exemple pour restaurer cette confiance.

\subsubsection{Le rôle de l'État}

Les réseaux électriques vont-ils disparaître, avec l'autonomie des producteurs territoriaux ? Que deviendra la péréquation nationale des tarifs lorsque chaque région sera autonome en énergie ? Comment la lutte contre la précarité énergétique sera-t-elle prise en compte?

Si les réseaux disparaissent, il n'y a plus besoin de régulateur, et la notion de service public aura vécu.

Selon que l'on optera ou non pour une taxe carbone, une tarification à la puissance ou à l'énergie, le maintien de la péréquation nationale des tarifs ou son abandon, le partage des coûts de la production d'électricité ne sera pas le même pour les consommateurs.

Ces questions et constats fait par Jacques Percebois n'ont pour l'instant pas de réponse ; car c'est à l'État, et pas seulement au marché qui a une vision de court terme, de dire ce qui est bon pour la collectivité sur le long terme. Mais les travaux et les avis de l'Office parlementaire des choix scientifiques et techniques (OPECST) restent pour le moment encore inconnus du grand public. Il est donc urgent de reprendre le chemin de la décarbonation de l'énergie sur des bases assainies, sans a priori ni objectifs irréalistes ; car nous, Français et Européens, avons une grande responsabilité vis-à-vis des pays en voie de développement dans la lutte contre le changement climatique.

\subsection{Définition des acronymes et abréviations utilisés dans le présent chapitre}

Black-out : Disruption générale du réseau électrique

$\mathrm{CO}_{2}$ : Gaz carbonique (gaz à effet de serre servant de référence, compte tenu de sa demi-vie dans l'atmosphère)

CSPE : $\quad$ Contribution au service public de l'électricité

EnRi : Énergie renouvelable intermittente (en pratique actuellement : éolien et solaire photovoltaïque)

$\mathrm{H}$ : $\quad$ Homme, ou Habitant

IDH : $\quad$ Indice de développement humain

LTECV : $\quad$ Loi n ${ }^{\circ}$ 2015-992 du 17 août 2015 relative à la Transition énergétique pour la croissance verte 
Mtep : million de tonne-équivalent pétrole (soit 11,6 TWh)

MWh, TWh: mégawatt-heure $(=1000 \mathrm{kWh})$; térawatt-heure $(1$ milliard de $\mathrm{kWh}$ )

NOME : Loi " Nouvelle organisation du marché de l'électricité "

PIB : $\quad$ Produit intérieur brut

PPE : Planification pluriannuelle de l'énergie (plan quinquennal de mise en application de la loi de transition énergétique)

RTE : $\quad$ Réseau de transport de l'électricité (haute tension)

Tep : $\quad$ Tonne-équivalent pétrole (soit 11,6 MW/h)

TRE : $\quad$ Taux de retour énergétique (Énergie brute/Énergie investie)

TRI : $\quad$ Temps de retour sur investissement

PV: $\quad$ (Solaire) photovoltaïque

\subsection{Références}

[1] Dominique Grenêche (Conférence).

[2] Rapport Énergies 2050, Centre d'analyses stratégiques, Premier ministre, Rapport du groupe de travail présidé par Jacques Percebois, 2012.

[3] Transition énergétique et (dé)croissance économique. Regards économiques. Institut de recherches économiques et sociales (Université catholique de Louvain), novembre 2017, $\mathrm{n}^{\circ} 135$.

[4] http://www.bretagne.developpement_durable.gouv.fr/IMG/pdf/ETUDE_ SOCIO_SRE_web2_cle74161a.pdf

[5] https://www.lesechos.fr/finance-marches/marches-financiers/030454538803metaux-les-besoins-colossaux-de-la-transition-energetique-2103122.php \#xtor=EPR-7-\%5Bmatinale\%5D-20170720-\%5BProv_\%5D-2051043 \%402

[6] René Kleijn et al. Metal requirements for low-carbon power generation. Energy, vol. 36, nº 9, September 2011.

[7] Performance Énergétique du Bâtiment - Programme de rénovation thermique du parc existant 2015-2050 - The Shift Project - 23 juin 2013.

[8] Proposition de résolution présentée au Sénat, n 194 du 4 décembre 2013. 